\title{
"Markedly Elevated Serum Alkaline Phosphatase in A Pregnancy with Acute on Chronic Chorioamnionitiswith Encephalocele,MeningoceleAnd Hydrocephalus in Fetus."
}

\author{
Dr. Bharat Veer Manchanda ${ }^{1}$,Dr. Rochak Pandey ${ }^{2}$,Dr. EshnaGupta ${ }^{3}$,Dr. Rahul \\ Chauhan ${ }^{4}$,Dr. AkshitMittal ${ }^{5}$,Dr. Saloni Mehra ${ }^{6}$ \\ ${ }^{1}$ Assi. Professor Indeptof Medicine, Subharti Medical College, Meerut \\ ${ }^{2,4,5}$ Post Graduate In Dept Of Medicine ,Subharti Medical College, Meerut \\ ${ }^{3}$ Post Graduate In Dept Of Obs. \&Gynae,Subharti Medical College, Meerut \\ ${ }^{6}$ Post Graduate In Dept Of Surgery ,Subharti Medical College, Meerut
}

\begin{abstract}
In normal pregnancy, although maternal serum alkaline phosphatase increases and reaches the twofold of non-pregnant serum level, above this level, hepatic, renal, bone diseases and malignancies should be kept in mind. We report a case of 26-year-old pregnant woman with increased maternal serum level of alkaline phosphatase (10-fold). Patient was induced and delivered a female baby with encephalocele,meningocele and hydrocephalus. After delivery, maternal serum alkaline phosphatase activity returned to normal reference range.
\end{abstract}

\section{Introduction}

Alkaline phosphatase (ALP, ALKP, ALPase, AlkPhos) is a hydrolase enzyme responsible for removing phosphate groups from many types of molecules, including nucleotides, proteins, and alkaloids. In humans, alkaline phosphatase is present in all tissues throughout the entire body, but is particularly concentrated in the liver,bileduct,kidneys,bones, intestinal mucosa and placenta Humans and most other mammals contain the following alkaline phosphatase isozymes:

ALPI-Intestinal

ALPL-Tissue non-specific (Liver/Bone/Kidney)

ALPP-Placental

Chorioamnionitis is a complication of pregnancy caused by bacterial infection of the fetal amnion and chorion membranes. Maternal chorioamnionitis occurs when protective mechanisms of the urogenital tract and/or uterus fail during pregnancy or when increased numbers of microbial flora or highly pathogenic microorganisms are introduced into the urogenital environment. Chorioamnionitis can occur with intact membranes, and this appears to be especially common for the very small fastidious genital mycoplasmas such as Ureaplasma species and Mycoplasma hominis, found in the lower genital tract of over $70 \%$ of women ${ }^{[1]}$. Only rarely is hematogeneous spread implicated in chorioamnionitis, as occurs with Listeria monocytogenes ${ }^{[2]}$. When characteristic clinical signs are present, the condition is referred to as clinical chorioamnionitis or clinical intraamniotic infection. Although there is significant overlap between clinical and histologic chorioamnionitis, the latter is a more common diagnosis based on pathologic findings on microscopic examination of the placenta that encompasses clinically unapparent (sub-clinical) chorioamnionitis as well as clinical chorioamnionitis.

\section{Case Report}

A 26 year old woman presented with gravida 4 para 2 live 3 abortion 1, for a routine prenatal visit at 36 weeks 0 days.The patient had come with the chief complaints of fever (101degree Farenheit) and reduced fetal movements for last 3 days.She had a past obstetric history of anencephaly 5 years back which was terminated. There was no history of any chronic illnesses like diabetes mellitus or hypothyroidism and no history of any drug intake.There was no history of bone,liver,kidney diseases or any malignancy. The general examination was suggestive of maternal tachycardia (120 beats/min).Systemic examination was unremarkable.The per abdominal examination was suggestive of relaxed uterus with a fundal height of 36 weeks and breechpresentation.The fetal heart sounds (FHS) were auscultated. The per speculum examination revelaed a healthy cervix and vagina.The per vaginal examination was suggestive of closed os and uneffacedcervix.The blood investigations were suggestive of maternal leukocytosis $\left(\right.$ TLC $11800 / \mathrm{mm}^{3}$ ). The liver function tests were normal except a markedly elevated alkaline phosphatase (1500 IU/L).The renal profile was normal.Urine routine examination revealed the presence of pus cells.

The ultrasonography revealed Biparietal Diameter (BPD) of $10.72 \mathrm{~cm}$,Head Circumference $(\mathrm{HC})$ of42.79 cm,Abdominal Circumference of $28.84 \mathrm{~cm}$,Amniotic Fluid Index (AFI) of 
$13 \mathrm{~cm}$, PosteriorPlacenta,,withBilateral Ventriculomegaly with Fetal hydrocephalus $(26 \times 27 \times 7 \mathrm{~mm})$ with occipital encephalocele with meningocele.

The histopathology of placenta was suggestive of Early iscahemia with Acute on Chronic Chorioamnionitis.Membranes showed marked inflammation in the form of neutrophils,lymphocytes and plasma cells.

\section{Discussion}

Risk factors for chorioamnionitis include obesity, diabetes, anemia, PPROM and the presence of genital tract pathogens ${ }^{[3]}$. It can be diagnosed clinically, pathologically or subclinically. Clinical diagnosis is based on the presence of maternal fever $\left(>38^{\circ} \mathrm{C}\right)$ and two of the following: maternal leukocytosis or leucopenia, maternal tachycardia (>100 bpm), fetal tachycardia (>160 bpm), uterine tenderness, and foul-smelling amniotic fluid $^{[4]}$.. Histological diagnosis is based on the identification of polymorphonuclear leucocytes on pathologic examination of the placenta and fetal membranes .Adverse maternal outcomes include postpartum infections and sepsis while adverse infant outcomes include stillbirth, premature birth, neonatal sepsis, chronic lung disease and brain injury leading to cerebral palsy and other neurodevelopmental disabilities. Research in the last two decades has expanded our understanding of the mechanistic links between intraamniotic infection and preterm delivery as well as morbidities of preterm and term infants.Chorioamnionitis (clinical and histologic combined), complicates as many as $40-70 \%$ of preterm births with premature membrane rupture or spontaneous labor ${ }^{[6]}$ and $1-13 \%$ of term births ${ }^{[7-9]}$. Fetal exposure to infection may lead to fetal death, neonatal sepsis and numerous other postnatal complications. The fetal response to infection - termed the Fetal Inflammatory Response Syndrome (FIRS) - may cause or aggravate some of these complications. chorioamnionitis is associated with up to $40 \%$ of cases of early-onset neonatal sepsis. Chorioamnionitis is also well-established as a risk factor for long-term neurodevelopmental disability especially when it occurs prior to term . In term and near-term infants it is associated with a 4 -fold increase in the frequency of cerebral palsy ${ }^{[10-11]}$

In our case the diagnosis was established on the basis of Maternal fever,maternaltachycardia,maternal leukocytosis and histopathological report of placenta.Chorioamnionitis has not been widely associated with congenital fetal anamolies and hence the authors believe this case to be of medical and obstetricsignificance.

\section{References}

[1]. Eschenbach DA. Ureaplasmaurealyticum and premature birth. Clin Infect Dis. 1993;17(Suppl 1):S100-6

[2]. 2. Gibbs RS, Duff P. Progress in pathogenesis and management of clinical intraamniotic infection. Am J Obstet Gynecol. $1991 ; 164: 1317$.

[3]. 3.Bacterial Sepsis in pregnancy. RCOG guideline 64A. April 2012

[4]. .Newton ER. Preterm labor, preterm premature rupture of membranes, and chorioamnionitis. ClinPerinatol. 2005;32:571-600)

[5]. Van Hoeven KH, Anyaegbunam A, Hochster H, et al. Clinical significance of increasing histologic severity of acute inflammation in the fetal membranes and umbilical cord. PediatrPathol Lab Med 1996;16:731-44.

[6]. Yoon BH, Romero R, Moon JB, et al. Clinical significance of intra-amniotic inflammation in patients with preterm labor and intact membranes. Am J Obstet Gynecol. 2001;185:1130.

[7]. Alexander JM, McIntire DM, Leveno KJ. Chorioamnionitis and the prognosis for term infants. Obstet Gynecol. 1999;94(2):274-8.

[8]. Seong HS, Lee SE, Kang JH, Romero R, Yoon BH. The frequency of microbial invasion of the amniotic cavity and histologic chorioamnionitis in women at term with intact membranes in the presence or absence of labor. Am J Obstet Gynecol. 2008;199(4):375.e1-5

[9]. Blume HK, Li CI, Loch CM, Koepsell TD. Intrapartum fever and chorioamnionitis as risks for encephalopathy in term newborns: a case-control study. Dev Med Child Neurol. 2008;50(1):19-24

[10]. Wu YW, Escobar GJ, Grether JK, et al. Chorioamnionitis and cerebral palsy in term and near-term infants. JAMA. 2003;290:2677.

[11]. Nelson KB, Ellenberg JH. Antecedents of cerebral palsy. I. Univariate analysis of risks. Am J Dis Child. 1985;139:1031. 